\title{
Regression of Corneal Vascularization Occurring after Corneal Suturing in a Case of Acute Hydrops Managed with a Combination Therapy of Argon Laser Photocoagulation and Intrastromal Ranibizumab Injection: A Case Report
}

\author{
Abraham Kurian ${ }^{1}$, Iodine Reghunadhan², Manoj Soman ${ }^{3}$, Mohammed Shahbaaz $^{4}$, Unni Nair ${ }^{5}$
}

\begin{abstract}
Aim: To report a case of acute hydrops with a large stromal cleft, in a case of keratoconus, managed with compression sutures and perfluoropropane (C3F8) descemetopexy and subsequent management of corneal vascularization in that eye with combination therapy of laser and anti-vascular endothelial growth factor (anti-VEGF).

Background: Acute hydrops in keratoconus occurs following a tear or rupture in the Descemet's membrane (DM), resulting in aqueous percolating into the stroma. The presence of large stromal clefts in acute hydrops has been described as a known risk factor for delayed resolution and persistent edema, which in turn can incite inflammation and vascularization and thereby adversely affect the chances of graft survival later. We describe such a case managed effectively with a combination of different treatment modalities.

Case description: A 12-year-old boy with keratoconus presented with acute hydrops in his left eye. The acute hydrops was managed with compression sutures along with C3F8 descemetopexy. Subsequent development of persistent deep corneal vascularization and recurrence of inflammation after the resolution of hydrops was managed with a combination of argon laser photocoagulation and intrastromal anti-VEGF injections, resulting in complete regression of the deep vascularization and resolution of inflammation.

Conclusion: In our case, a combination of management modalities to address severe acute hydrops and its subsequent complications resulted in complete regression of the superficial and deep vessels, thus heralding an optimal outcome for a future corneal graft.

Clinical significance: There are no definite guidelines for the management of acute hydrops in keratoconus. A customized and judicious combination of various treatment modalities described in the literature for acute hydrops and its sequelae can result in an accepted outcome, which can pave the way for an optimum result with elective treatments like keratoplasty later on.
\end{abstract}

Keywords: Acute hydrops, Case report, Corneal vascularization, Keratoconus.

International Journal of Keratoconus and Ectatic Corneal Diseases (2019): 10.5005/jp-journals-10025-1182

\section{BACKGROUND}

Acute hydrops in keratoconus (KC) occurs following a tear or a rupture in the Descemet's membrane (DM), resulting in aqueous percolating into the stroma. The first case of hydrops in $\mathrm{KC}$ was reported by Plaut (1900) as a sudden opacity at the apex of the cornea. ${ }^{1,2}$ Acute hydrops occurs in approximately $2.5-3 \%$ of eyes with KC. ${ }^{1,3,4}$ The presence of large stromal clefts in acute hydrops has been described as a known risk factor for delayed resolution and persistent edema, which in turn can incite inflammation and vascularization and thereby adversely affect the chances of graft survival later. We describe such a case managed effectively with a combination of different treatment modalities.

\section{Case Description}

A 12-year-old Maldivian boy, a diagnosed case of KC, was brought with complaints of noticing a whitish discoloration of the left eye since three days, associated with occasional pain. He also gave a history of diminution of vision in both eyes for the past two years, which had become worse in his left eye, associated with the presenting complaints. He had no history of atopy or contact lens
${ }^{1}$ Department of Cornea, Orbit and Oculoplasty, Strabismus, Paediatric Ophthalmology, Chaithanya Eye Hospital and Research Institute, Trivandrum, Kerala, India

${ }^{2}$ Department of Comprehensive Ophthalmology, Chaithanya Eye Hospital and Research Institute, Trivandrum, Kerala, India

3,5 Department of Vitreoretinal Services, Chaithanya Eye Hospital and Research Institute, Trivandrum, Kerala, India

${ }^{4}$ Department of Cornea, Chaithanya Eye Hospital and Research Institute, Trivandrum, Kerala, India

Corresponding Author: Abraham Kurian, Department of Cornea, Orbit and Oculoplasty, Strabismus, Paediatric Ophthalmology, Chaithanya Eye Hospital and Research Institute, Trivandrum, Kerala, India, Phone: +91 9847094478, e-mail: abrahamkurian55@yahoo.in

How to cite this article: Kurian A, Reghunadhan I, Soman M, et al. Regression of Corneal Vascularization Occurring after Corneal Suturing in a Case of Acute Hydrops Managed with a Combination Therapy of Argon Laser Photocoagulation and Intrastromal Ranibizumab Injection: A Case Report. Int J Kerat Ect Cor Dis 2019;8(2):46-49.

Source of support: Nil

Conflict of interest: None

(c) The Author(s). 2019 Open Access This article is distributed under the terms of the Creative Commons Attribution 4.0 International License (https:// creativecommons.org/licenses/by-nc/4.0/), which permits unrestricted use, distribution, and non-commercial reproduction in any medium, provided you give appropriate credit to the original author(s) and the source, provide a link to the Creative Commons license, and indicate if changes were made. The Creative Commons Public Domain Dedication waiver (http://creativecommons.org/publicdomain/zero/1.0/) applies to the data made available in this article, unless otherwise stated. 

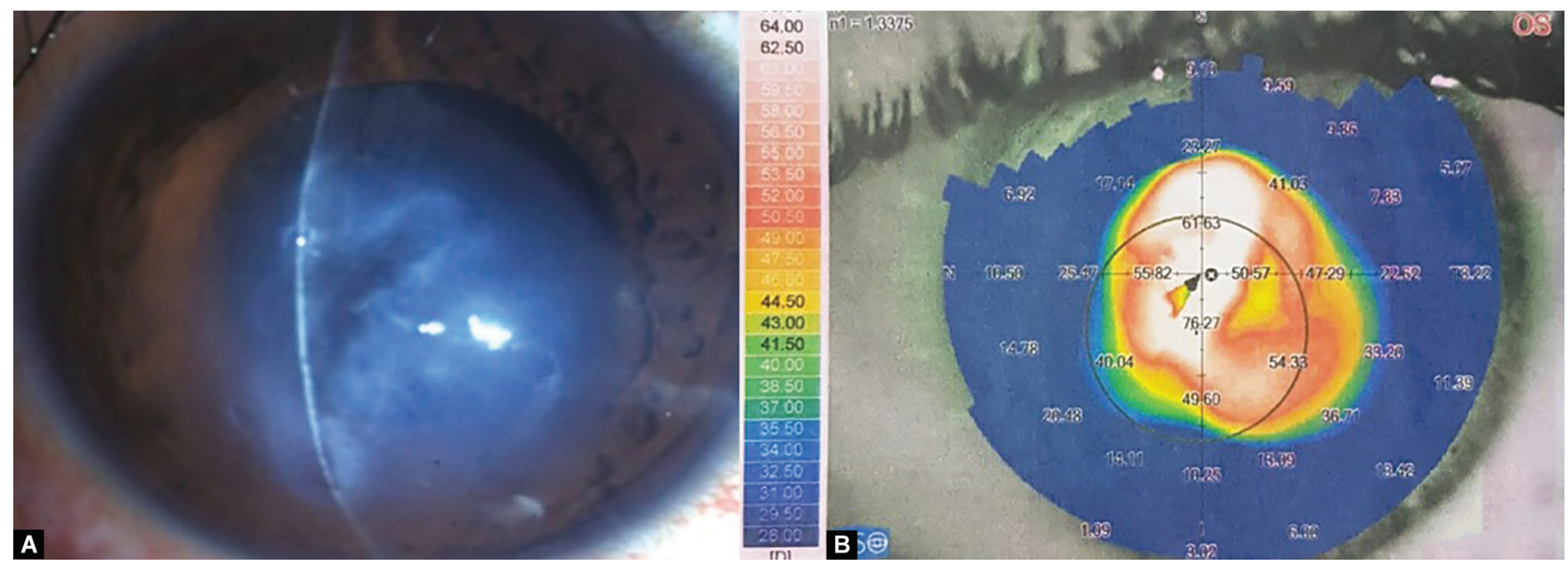

Figs 1 A and B: (A) Clinical photograph at presentation-acute hydrops. (B) Corneal topography of the eye with acute hydrops showing gross central steepening with irregular astigmatism
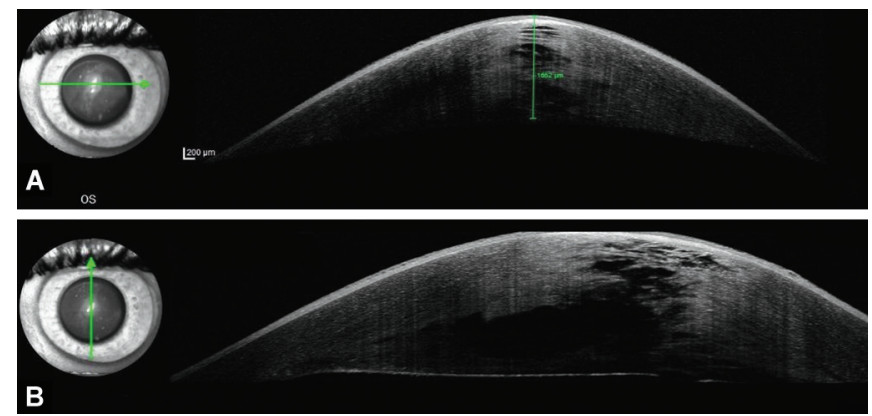

Figs $2 \mathrm{~A}$ and B: ASOCT of the left eye showing a huge stromal cleft with an area of discontinuity in the DM. The central corneal thickness is measured as $1652 \mu \mathrm{m}$

use. His vision was $6 / 18$ in the right eye and counting of fingers close to face in the left eye, not improving with a pin hole. External eye examination was clinically normal in the right eye and was suggestive of acute hydrops in the left eye (Fig. 1A).

Corneal topography was suggestive of $\mathrm{KC}$ in the right eye with the thinnest pachymetry of $450 \mu \mathrm{m}$. Topography of the left eye showed gross central steepening with irregular astigmatism (Fig. 1B).

An anterior segment optical coherence tomography (ASOCT) of the left eye showed a huge stromal cleft with an area of discontinuity in the DM. The central corneal thickness was measured as $1652 \mu \mathrm{m}$ (Figs 2A and 2B).

He underwent corneal collagen cross-linking with isotonic riboflavin in the right eye. For the left eye, full-thickness corneal sutures were applied in the area of the stromal cleft after an injection of $14 \%$ C3F8. His immediate postoperative period was uneventful. Two weeks later, the corneal edema subsided and his uncorrected vision in the left eye had improved to $6 / 36$. Two months postoperatively, he developed vascularization of the cornea with a leash of deep vessels, extending to about $5 \mathrm{~mm}$ into the cornea to the superior end of the scar from the 11 o'clock limbus at the superior end of the sutured wound which had healed. Subsequently, he underwent removal of the corneal sutures.
Later, at five months postoperatively, he presented with redness and watering in the right eye and the examination showed persistence of the deep corneal leash of the vessels, still extending to about $5 \mathrm{~mm}$ into the cornea to the superior end of the scar from the 11 o'clock limbus, with mild stromal edema and anterior uveitis (Figs 3A and 3B). His vision in that eye had dropped to count finger (CF) vision at $3 \mathrm{~m}$.

He was started on topical steroids and lubricants. In view of the deep vascularization, direct laser photocoagulation to the deep leash of vessels was attempted with argon laser using the following settings $-75 \mu$ spot size, $100 \mathrm{~ms}$ and $500 \mathrm{mw}$, which resulted in some blanching of the large blood vessel. However, the small vessels were not taking up the laser burns. This was combined with an intrastromal injection of $0.5 \mathrm{mg} / 0.05 \mathrm{ml}$ of ranibizumab the next day. Two days following the injection, the vessels started showing regression with blanching of the edges at the limbus.

One month after the procedure, both the large and small vessels had regressed completely and the anterior segment inflammation and stromal edema had also subsided (Fig. 4).

His vision reverted to $6 / 36$ as was before the development of vascularization. He underwent one more intrastromal injection of ranibizumab and is being planned for an elective optical keratoplasty.

\section{Discussion}

Conventional therapy for acute hydrops is patching or therapeutic bandage contact lens application with topical cycloplegics and topical hypertonic sodium chloride. ${ }^{5}$ However, during recovery, patient suffers from severe photophobia and irritation. Spontaneous resolution usually occurs by endothelial sliding over a period of 2-4 months. ${ }^{5}$ It has been reported in previous case reports that resolution of acute hydrops in its natural course results in extreme thinning and flattening of the cornea, ${ }^{6}$ which can in turn compromise the tectonic integrity of the globe and adversely affect the subsequently planned elective procedures for tectonic and/or optical rehabilitation.

The Descemet's rupture in acute hydrops leads to percolation of aqueous and separation of the collagen lamellae, resulting in large fluid-filled stromal clefts. In some cases, the presence of large stromal clefts delays resolution and the persistent edema can incite inflammation and vascularization that may adversely affect the chances of graft survival later. ${ }^{6}$ Intracameral injection of air or 

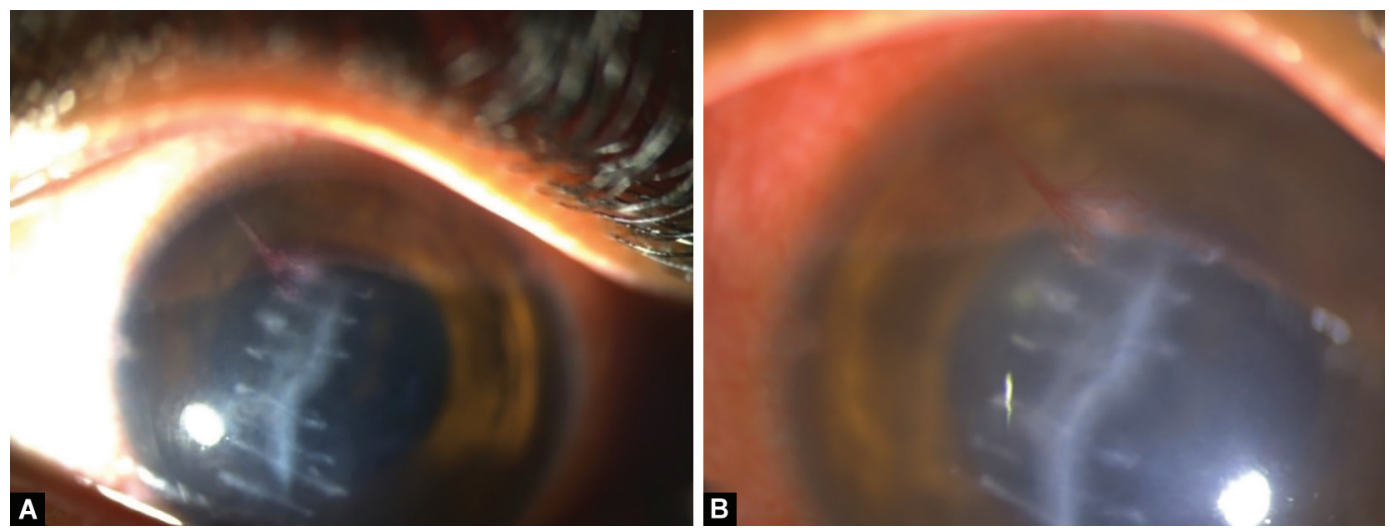

Figs $3 \mathrm{~A}$ and B: Persistent deep corneal vascularization extending to about $5 \mathrm{~mm}$ into the cornea to the superior end of the scar from the 11 o'clock limbus with mild stromal edema and anterior uveitis

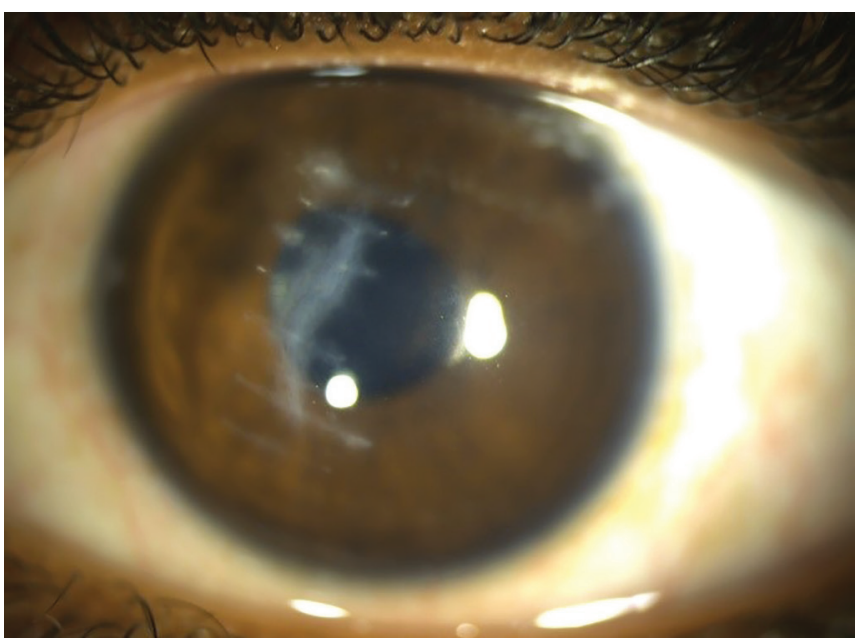

Fig. 4: Complete regression of the deep vascularization, inflammation, and stromal edema

nonexpansile gas mixtures shortens the recovery period by blocking the entry of aqueous into the stroma by a tamponade effect and also unrolls the torn ends of the ruptured DM. ${ }^{5,7}$ Various agents used include air, 20\% sulfur hexafluoride (SF6), and 14\% perfluoropropane (C3F8). ${ }^{8}$ Air stays for a shorter time, hence repeat injections may be required. ${ }^{8}$ SF6 is long acting compared to air (around two weeks); however, repeat injections may still be required. ${ }^{8} \mathrm{C} 3 \mathrm{~F} 8$ is the longest acting among all and usually repeat injections are not required. ${ }^{1,3,5,8}$

Other surgical modalities that are helpful in special situations complicating hydrops include compressive sutures along with gas injection, ${ }^{7}$ Penetrating keratoplasty, ${ }^{1}$ cyanoacrylate tissue adhesive with bandage contact lens, ${ }^{8}$ and amniotic membrane transplantation with cauterization. ${ }^{8}$

Compressive sutures along with gas injection have been tried in severe cases with wide separation of the DM edges and multiple stromal clefts. ${ }^{1,7}$

Considering the huge stromal clefts in this case and widely separated edges of the DM along most of the involved area, a combination of full-thickness sutures and C3F8 descemetopexy was deemed optimum to hasten the resolution of the hydrops. Following this, the hydrops resolved in 2 weeks. But, however, the presence of deep neovascularization was noticed at the 2-month review and the corneal sutures were removed, considering them to be the continuing risk factor to incite vascularization and inflammation as the hydrops had resolved. In spite of removing all the sutures at 5 months postoperatively, he presented with recurrence of inflammation and edema and persistence of deep corneal vascularization. Considering this to be a risk factor that would compromise the corneal clarity and predispose to graft rejection by breaching the immune privilege of the cornea after a future keratoplasty, the options to tackle the vascularization were analyzed.

Since VEGF plays an important role in the pathologic neovascularization in a huge number of eye diseases hence, it is the most important target for antiangiogenic treatments. ${ }^{9}$ According to the literature, anti-VEGF agents are quite effective in occluding the actively growing blood vessels but not establishing large vessels in which surgical approaches, such as fine needle diathermy or laser photocoagulation, are invaluable. ${ }^{9}$

Laser photocoagulation is a simple outpatient procedure and both argon laser and 577-nm yellow dye laser have been described to effectively obliterate the efferent corneal blood vessels as these wide vessels have a relatively slower blood flow. ${ }^{9,10}$ On the contrary, it is more difficult to obliterate the afferent vessels because these vessels are thinner and deeper and have a fast blood flow. ${ }^{9,10}$ Thus, reopening of the treated afferent vessels occurs in a high percentage of cases, necessitating multiple treatments. However, in cases with extensive corneal neovascularization, laser photocoagulation may be ineffective. ${ }^{10}$

\section{Conclusion}

In our case, a combination of management modalities to address severe acute hydrops and its subsequent complications resulted in complete regression of the superficial and deep vessels, thus heralding an optimal outcome for a future corneal graft.

\section{Clinical Significance}

There are no definite guidelines for the management of acute hydrops in KC. A customized and judicious combination of various treatment modalities described in the literature for acute hydrops and its sequelae can result in an accepted outcome, which can pave the way for an optimum result with elective treatments like keratoplasty later on. 


\section{References}

1. Tuft SJ, Gregory WM, Buckley RJ. Acute corneal hydrops in keratoconus. Ophthalmology 1994;101(10):1738-1744. DOI: 10.1016/ s0161-6420(94)31110-9.

2. Plaut R. Ueber Verdickung der Hornhaut beim Keratoconus. Klin Monatsbl Augenheilkd 1900;38:65-83.

3. Basu S, Vaddavalli PK, Ramappa M, et al. Intracameral perfluoropropane gas in the treatment of acute corneal hydrops. Ophthalmology 2011;118(5):934-939. DOI: 10.1016/j.ophtha.2010. 09.030 .

4. Grewal S, Laibson PR, Cohen EJ, et al. Acute hydrops in the corneal ectasias: associated factors and outcomes. Trans Am Ophthalmol Soc 1999;97:187-203.

5. Srirampur A, Kalwad A. Pneumodesmetopexy with perfluoropropane for acute corneal hydrops in keratoconus. Oman J Ophthalmol 2019;12(1):65-66. DOI: 10.4103/ojo.0JO_30_2018.
6. Loh I-P, Fan Gaskin JC, Sherwin T, et al. Extreme Descemet's membrane rupture with hydrops in keratoconus: clinical and histological manifestations. Am J Ophthalmol Case Rep 2018 Apr;10:271-275. DOI: 10.1016/j.ajoc.2018.04.003.

7. Rajaraman $R$, Singh $S$, Raghavan A, et al. Efficacy and safety of intracameral perfluoropropane (C3F8) tamponade and compression sutures for the management of acute corneal hydrops. Cornea 2009;28(3):317-320. DOI: 10.1097//CO.0b013e31818ada0b.

8. Maharana PK, Sharma N, Vajpayee RB. Acute corneal hydrops in keratoconus. Indian J Ophthalmol 2013 Aug;61(8):461-464. DOI: 10.4103/0301-4738.116062.

9. Feizi S, Azari AA, Safapour S. Therapeutic approaches for corneal neovascularization. Eye Vis (Lond). 2017;4:28. DOI: 10.1186/s40662017-0094-6.

10. Baer JC, Foster CS. Corneal laser photocoagulation for treatment of neovascularization. Efficacy of $577 \mathrm{~nm}$ yellow dye laser. Ophthalmology 1992;99:173-179. 